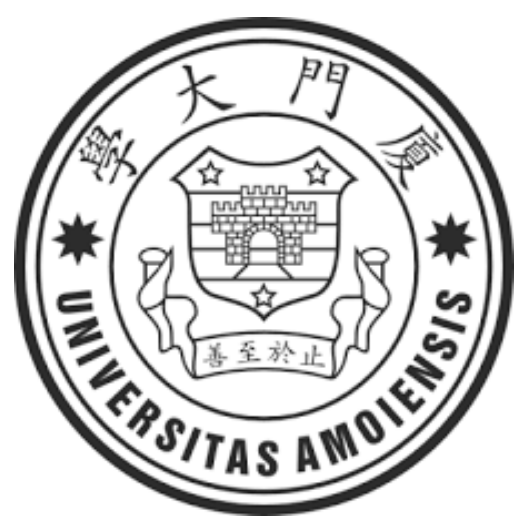

Cyber-bullying among Traditional and Complementary Medicine Practitioners at workplace cross-sectional descriptive study

Workplace Cyber-bullying

Title of Research: Cyber-bullying among Traditional and Complementary Medicine Practitioners at workplace: A cross-sectional descriptive study

Principal Investigator: Dr. Muhammad Shahzad Aslam

Co-Investigator: Dr. Yun Jin Kim; Dr. Linchao Qian

Funding: Research Management Center, Xiamen University Malaysia (XMUMRF/2020-C6/ITCM/0005) What is the objective of Study?

The purpose of this survey is to investigate workplace cyber-bullying on Traditional and Complementary Medicine Practitioners in Malaysia. This is a research project being conducted by Dr. Muhammad Shahzad Aslam, School of Traditional Chinese Medicine at Xiamen University Malaysia.

Who Can take part in this study?

Traditional and Complementary Medicine Practitioners (TCMPs) working in public sector, private sector in Malaysia. The participants from all recognized practice areas by Ministry of health Malaysia (Traditional Malay Medicine, Traditional China Medicine, Traditional India Medicine, Homeopathy, Chiropractic, Osteopathy and Islamic medical practice) are included in the study. Participants in this online survey is voluntary. You may choose not to participate. If you decide to participate in this online survey, you may withdraw at any time. If you decide not to participate in this online survey or if you withdrawal from participating at any time, you will not be penalized.

\title{
Procedure:
}

The procedure involves filling an online surveys will take approximately 15-20 minutes. Your responses will be confidential and we do not collect identifying information such as your name, IC Number, Contact number or IP address. The survey questions will be about to ask from Traditional and Complementary Medicine Practitioners on cyberbullying behaviour.

We will do our best to keep your information confidential. All data is stored in a password protected electronic format. To help protect your confidentiality, the survey will not contain information that will personally identify you. The results of this study will be used for research purposes only.

If you have any questions about the research study, please contact to Dr. Muhammad Shahzad Aslam (aslam.shahzad@xmu.edu.my)

Thank you so much and take a good care. 


\section{Best regards}

\section{Dr. Muhammad Shahzad Aslam}

\section{Xiamen University Malaysia}

Version: Questionaire_Aslam_V0.2_22November2020

* 1. I agree that any personal information provided in this online survey can be used for the purposes(s) mentioned in the privacy Notice.
Agree
Disagree

*2. Practice Area

Traditional Malay Medicine

Traditional China Medicine

Traditional India Medicine

Homeopathy

Chiropractic

Osteopathy

Islamic medical practice

Other than recognized practice areas by Ministry of health Malaysia

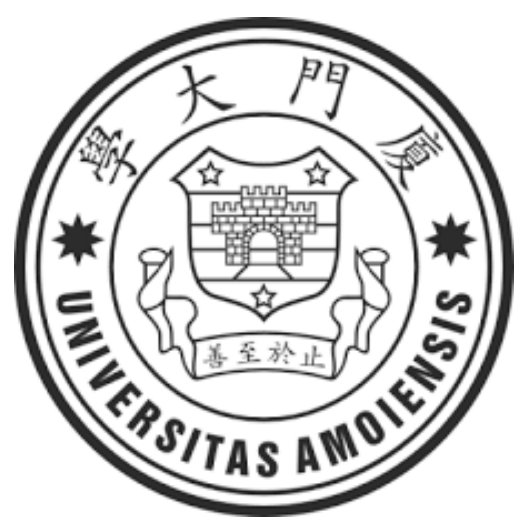

Cyber-bullying among Traditional and Complementary Medicine Practitioners at workplace cross-sectional descriptive study

Email address and their Registration number from Official governing bodies Your information will kept confidential and only need for verification purpose 
* 3. Experience in relevant field (in years)
1
2
3
4
5
$5+$

* 4. Working Sector

Government Hospital

Government Health Clinic

Private Hospitals

Private Clinic

Other

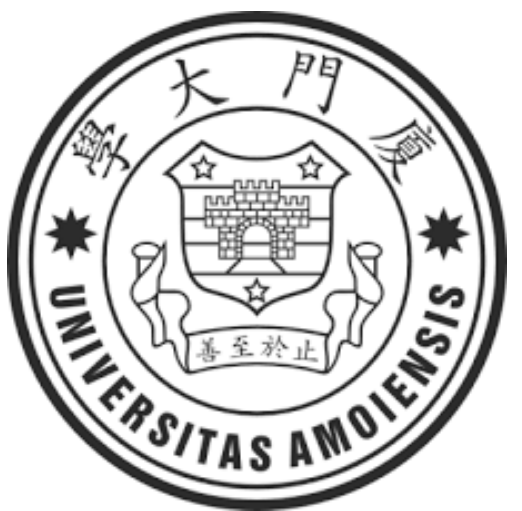

Cyber-bullying among Traditional and Complementary Medicine Practitioners at workplace cross-sectional descriptive study

* 5. Your work performance has been commented upon in negative terms on the Internet

Strongly agree

Agree

Neither agree nor disagree

Disagree

Strongly disagree 


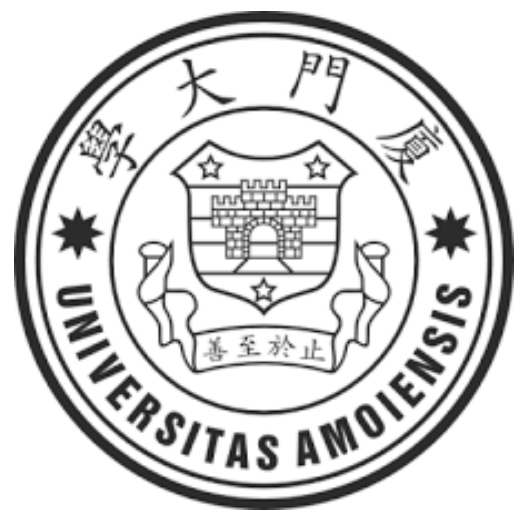

Cyber-bullying among Traditional and Complementary Medicine Practitioners at workplace cross-sectional descriptive study

*6. Gossip or rumors about you have been spread on the Internet

Strongly agree

Agree

Neither agree nor disagree

Disagree

Strongly disagree

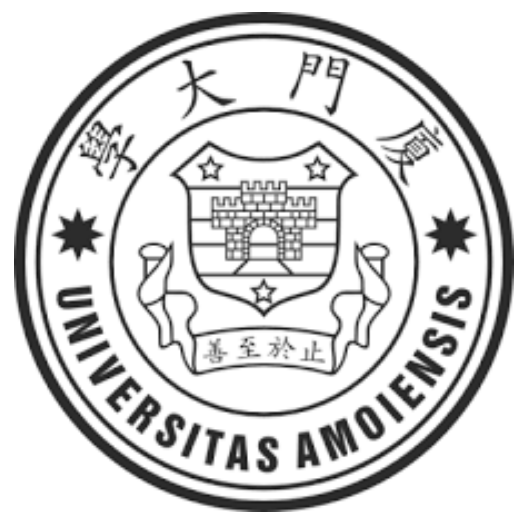

Cyber-bullying among Traditional and Complementary Medicine Practitioners at workplace cross-sectional descriptive study

* 7. In what type of community do you work?

City or urban community

Suburban community

Rural community

Other (please specify) 


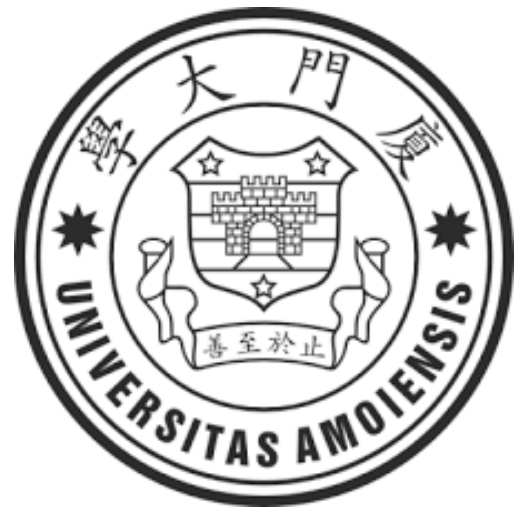

Cyber-bullying among Traditional and Complementary Medicine Practitioners at workplace cross-sectional descriptive study

* 8. Threatening personal messages have been sent to you via digital media

Strongly agree

Agree

Neither agree nor disagree

Disagree

Strongly disagree

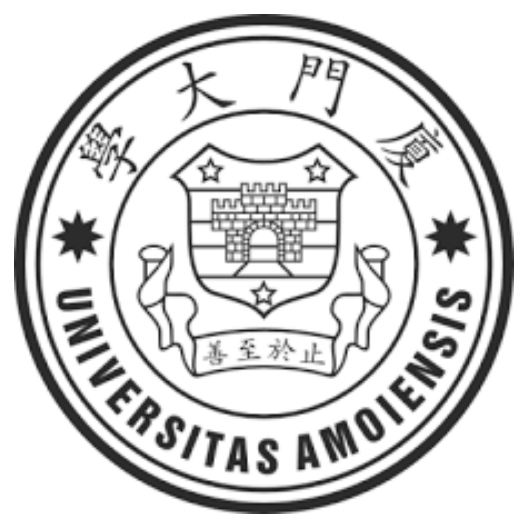

Cyber-bullying among Traditional and Complementary Medicine Practitioners at workplace cross-sectional descriptive study

* 9. Persistent criticism of your work or performance has been made against you via digital media

Strongly agree

Agree

Neither agree nor disagree

Disagree

Strongly disagree 


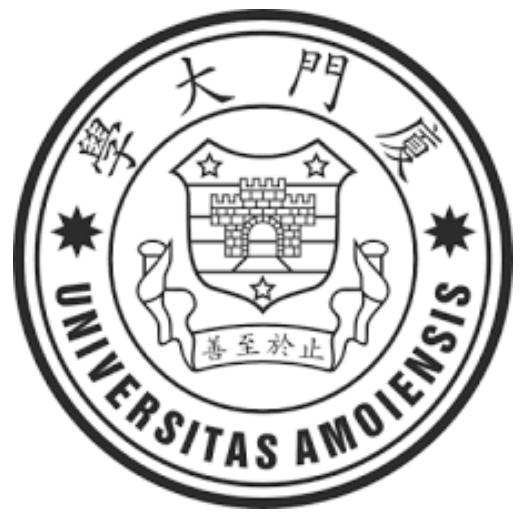

Cyber-bullying among Traditional and Complementary Medicine Practitioners at workplace cross-sectional descriptive study

* 10. Others have commented on the Internet that you should quit your work

Strongly agree

Agree

Neither agree nor disagree

Disagree

Strongly disagree

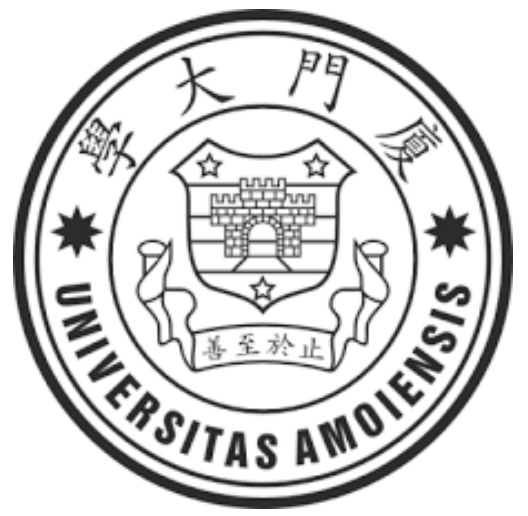

Cyber-bullying among Traditional and Complementary Medicine Practitioners at workplace cross-sectional descriptive study

* 11. Your computer identity has been hijacked

Strongly agree

Agree

Neither agree nor disagree

Disagree

Strongly disagree 


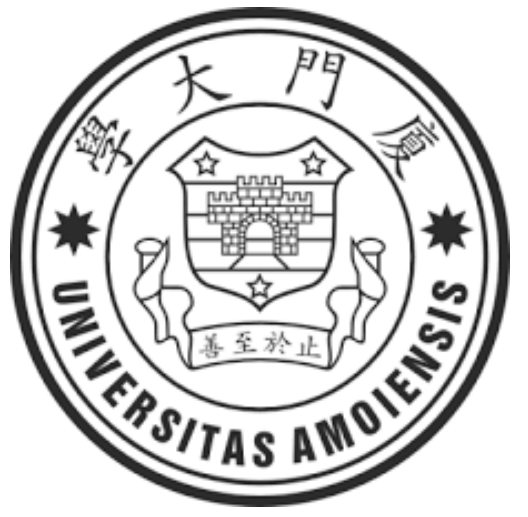

Cyber-bullying among Traditional and Complementary Medicine Practitioners at workplace cross-sectional descriptive study

* 12. Colleagues have excluded you from the social community online (e.g. Facebook, Twitter)

Strongly agree

Agree

Neither agree nor disagree

Disagree

Strongly disagree

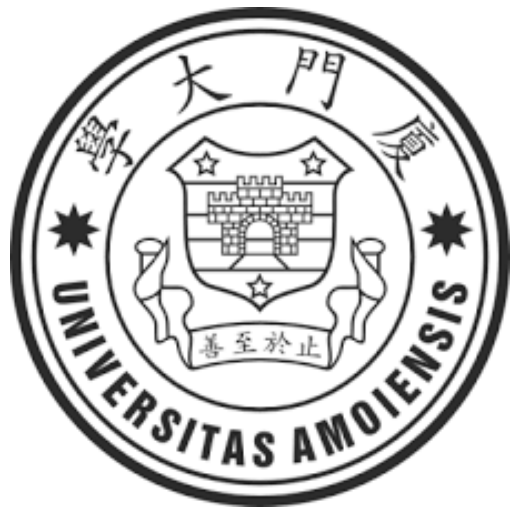

Cyber-bullying among Traditional and Complementary Medicine Practitioners at workplace cross-sectional descriptive study

* 13. Rude messages have been sent to you via digital media

Strongly agree

Agree

Neither agree nor disagree

Disagree

Strongly disagree 


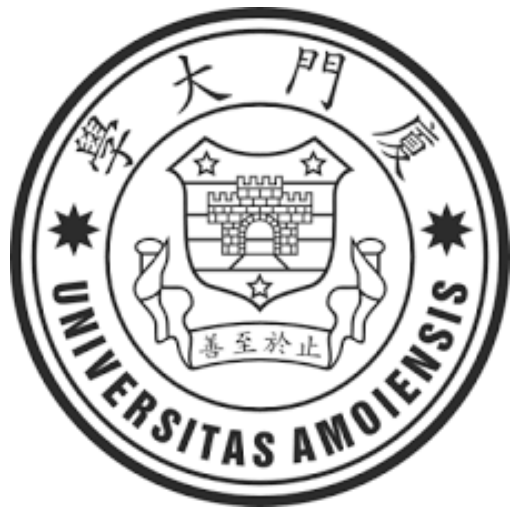

Cyber-bullying among Traditional and Complementary Medicine Practitioners at workplace cross-sectional descriptive study

* 14. Your mistakes or errors at work are repeatedly commented about in e-mails, text messages, or the like

Strongly agree

Agree

Neither agree nor disagree

Disagree

Strongly disagree

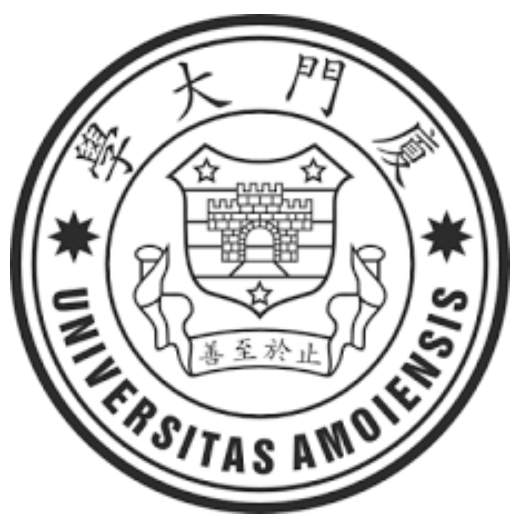

Cyber-bullying among Traditional and Complementary Medicine Practitioners at workplace cross-sectional descriptive study

* 15 . What is your sex?

Male

Female 


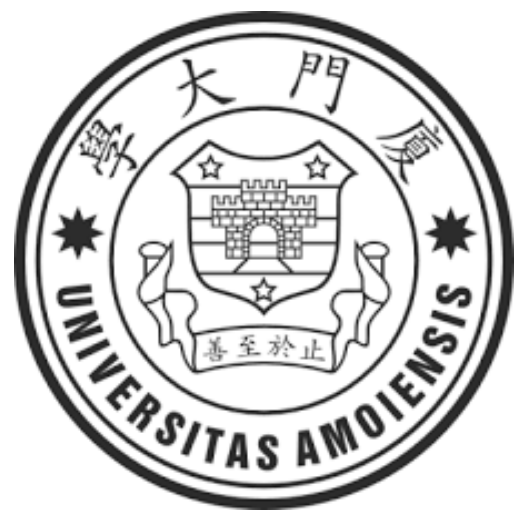

Cyber-bullying among Traditional and Complementary Medicine Practitioners at workplace cross-sectional descriptive study

\section{Age group}

$18-24$

$25-34$

$45-54$

$55-64$

$65+$

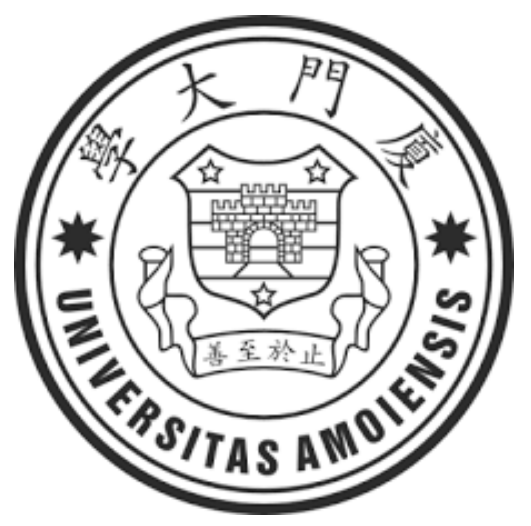

Cyber-bullying among Traditional and Complementary Medicine Practitioners at workplace cross-sectional descriptive study

* 17 . Viruses have intentionally been sent to your e-mail address

Strongly agree

Agree

Neither agree nor disagree

Disagree

Strongly disagree 


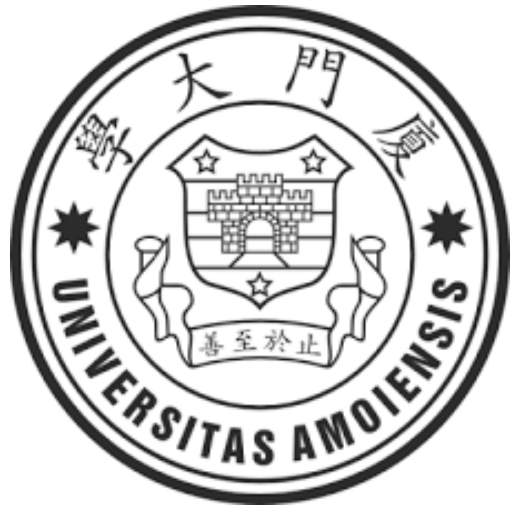

Cyber-bullying among Traditional and Complementary Medicine Practitioners at workplace cross-sectional descriptive study

* 18. Your supervisor/colleagues are not responding to your e-mails or text messages

Strongly agree

Agree

Neither agree nor disagree

Disagree

Strongly disagree

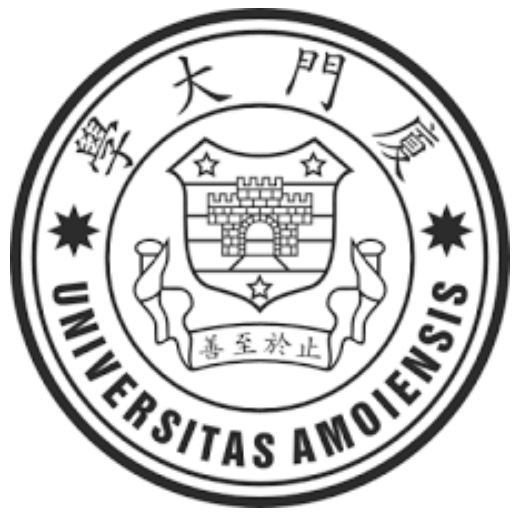

Cyber-bullying among Traditional and Complementary Medicine Practitioners at workplace cross-sectional descriptive study 
* 19. Extracts from your messages have been copied so that the meaning of the original message is distorted

Strongly agree

Agree

Neither agree nor disagree

Disagree

Strongly disagree

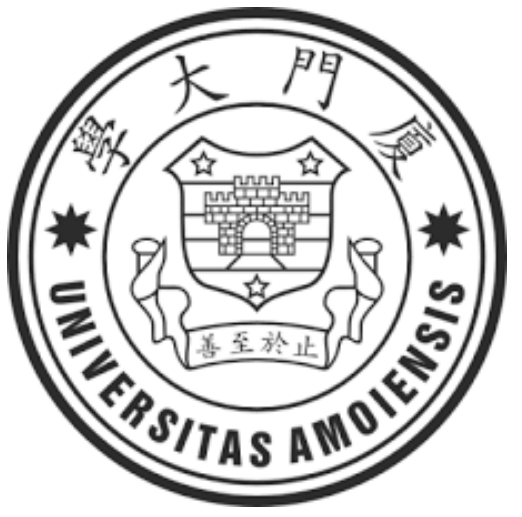

Cyber-bullying among Traditional and Complementary Medicine Practitioners at workplace cross-sectional descriptive study

* 20. Aggressively worded messages (e.g. capital letters, bold style or multiple exclamation marks) have been sent to you via e-mail, text messages)

Strongly agree

Agree

Neither agree nor disagree

Disagree

Strongly disagree

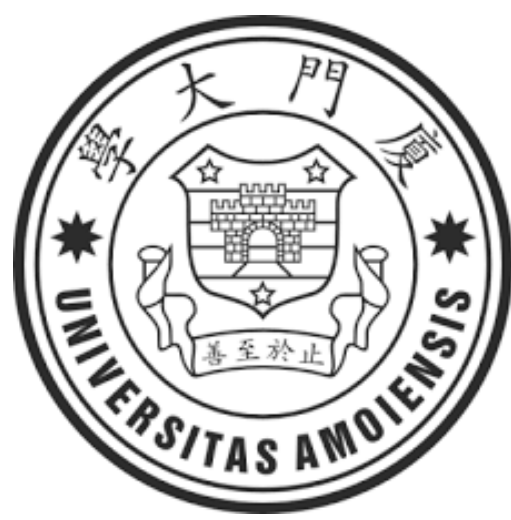


Cyber-bullying among Traditional and Complementary Medicine Practitioners at workplace cross-sectional descriptive study

* 21. What is the highest level of education you have completed?

Diploma

Professional degree

Undergraduate

Masters

Ph.D.

Other

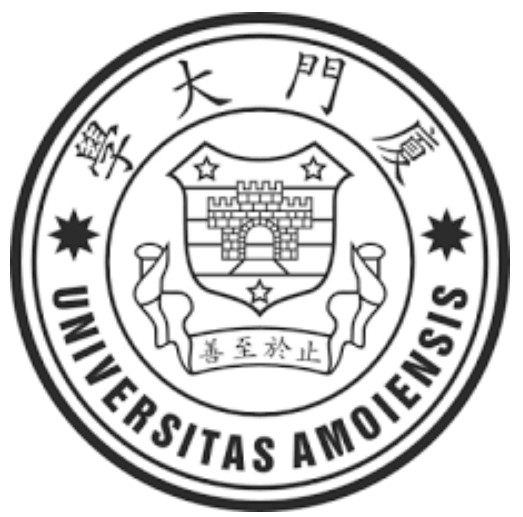

Cyber-bullying among Traditional and Complementary Medicine Practitioners at workplace cross-sectional descriptive study

* 22. Which of the following best describes your current relationship status?

Married

Widowed

Divorced

Separated

In a domestic partnership or civil union

Single, but cohabiting with a significant other

Single, never married 


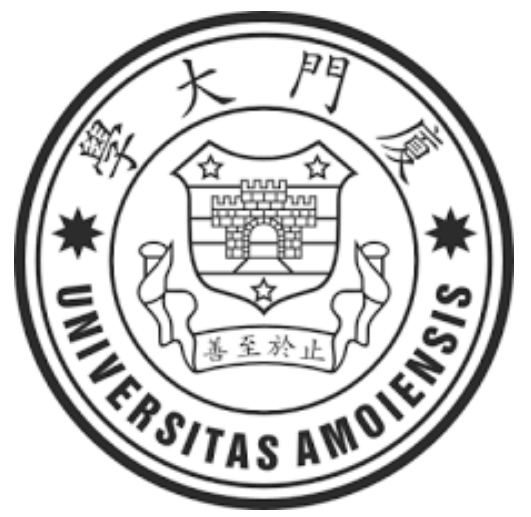

Cyber-bullying among Traditional and Complementary Medicine Practitioners at workplace cross-sectional descriptive study

* 23. Allegations about you have been made on the Internet

Strongly agree

Agree

Neither agree nor disagree

Disagree

Strongly disagree

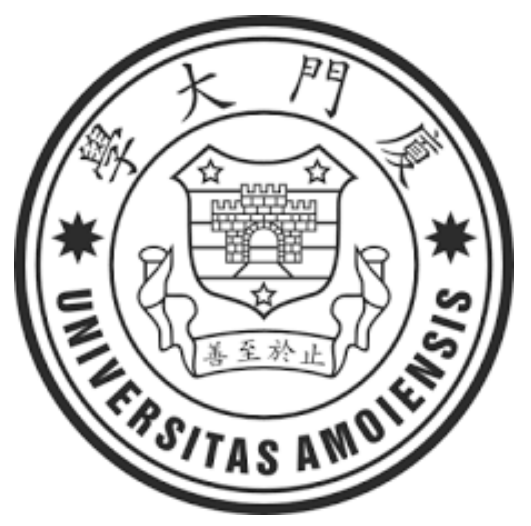

Cyber-bullying among Traditional and Complementary Medicine Practitioners at workplace cross-sectional descriptive study 
* 24. Attacks against you as a person, your values or your personal life have been made on digital media

Strongly agree

Agree

Neither agree nor disagree

Disagree

Strongly disagree

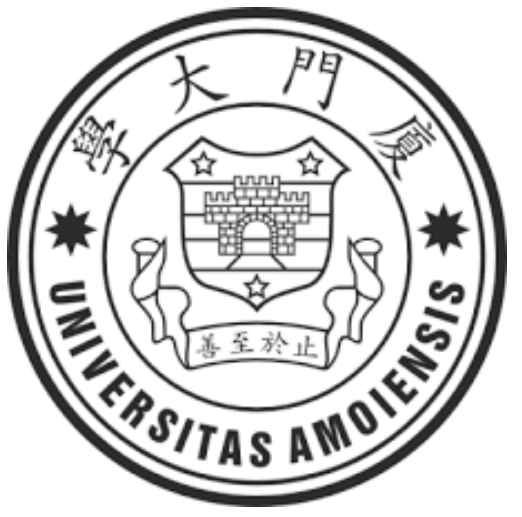

Cyber-bullying among Traditional and Complementary Medicine Practitioners at workplace cross-sectional descriptive study

* 25. False statements about you have been spread on the Internet

Strongly agree

Agree

Neither agree nor disagree

Disagree

Strongly disagree

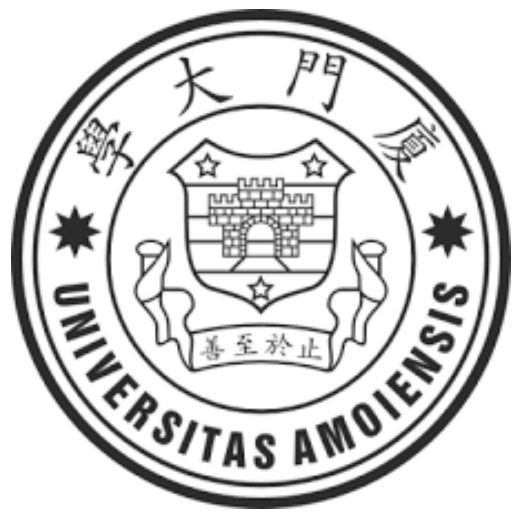


Cyber-bullying among Traditional and Complementary Medicine Practitioners at workplace cross-sectional descriptive study

* 26. Threatening messages about your friends/your family have been sent to you via digital media

Strongly agree

Agree

Neither agree nor disagree

Disagree

Strongly disagree

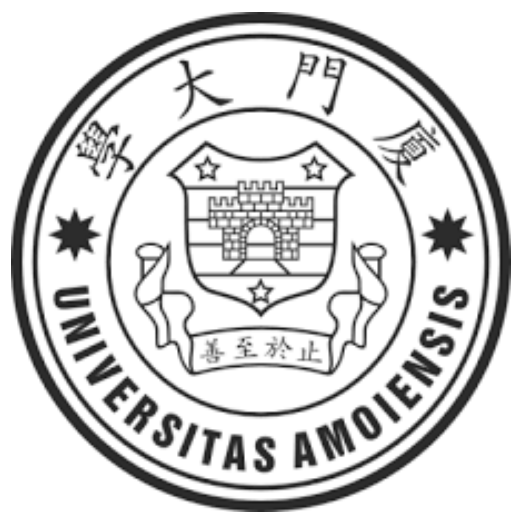

Cyber-bullying among Traditional and Complementary Medicine Practitioners at workplace cross-sectional descriptive study

*27. Jokes about you have been spread on the Internet or via e-mail to several recipients

Strongly agree

Agree

Neither agree nor disagree

Disagree

Strongly disagree 


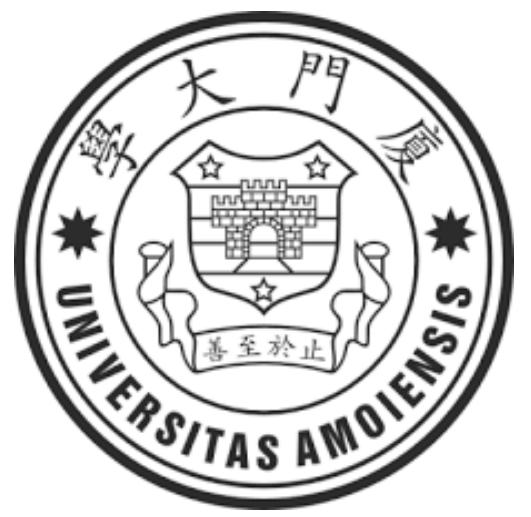

Cyber-bullying among Traditional and Complementary Medicine Practitioners at workplace cross-sectional descriptive study

* 28. Necessary information has been withheld making your work more difficult (e.g. being excluded from e-mail lists)

Strongly agree

Agree

Neither agree nor disagree

Disagree

Strongly disagree

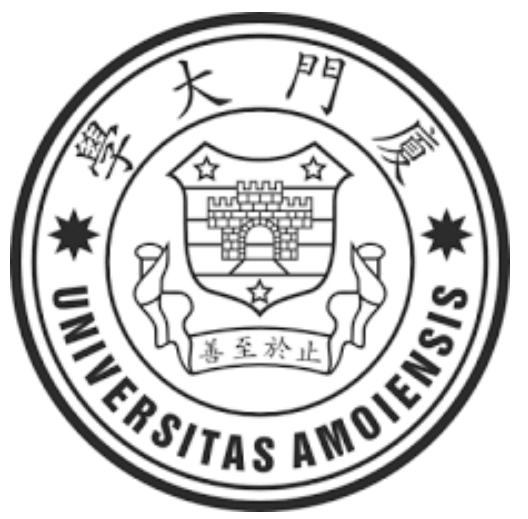

Cyber-bullying among Traditional and Complementary Medicine Practitioners at workplace cross-sectional descriptive study 
* 29. Offensive photos/videos of you have been posted on the Internet

Strongly agree

Agree

Neither agree nor disagree

Disagree

Strongly disagree

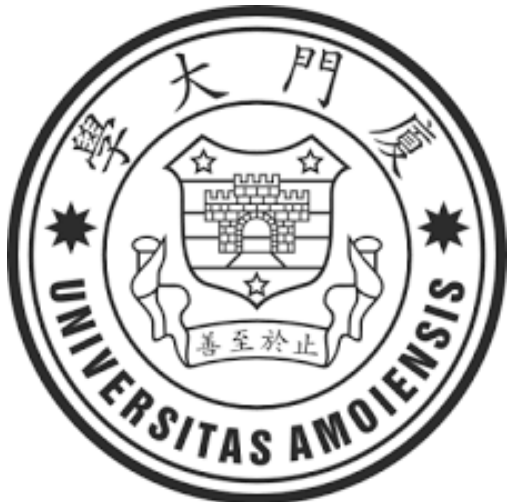

Cyber-bullying among Traditional and Complementary Medicine Practitioners at workplace cross-sectional descriptive study

* 30 . Which of the following best describes your current job level?

Owner/Executive/C-Level

Senior Management

Middle Management

Intermediate

Entry Level

Other (please specify)

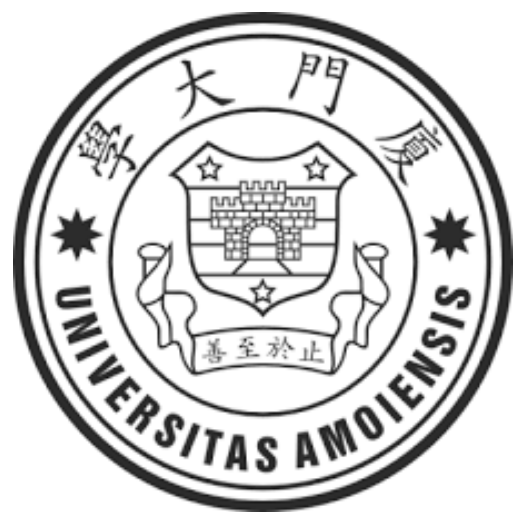


Cyber-bullying among Traditional and Complementary Medicine Practitioners at workplace cross-sectional descriptive study

* 31. City

Kualalumpur

Malacca

Penang

Kotakinabalu

Ipoh

Kuching

Johor Bahru

Kuantan

Alorsetar

Sandakan

Kangar

Kelantan

Other

* 32. What is your approximate average household income?

MYR 0-999

MYR 1,000-2,999

MYR 3,000-4,999

MYR 5,000-6,999

MYR 7,000-8,999

MYR 9,000-10,999

MYR 11,000-12,999

MYR 13,000 and up

* 33. Are you involve in Substance abuse such as Cigarette, Cigar, pipe, tobacco, alcohol, non-prescribed cough syrup or psychoactive pill?

Yes

No 